\title{
PRESCRIBED CURVATURE TENSOR IN LOCALLY CONFORMALLY FLAT MANIFOLDS
}

\author{
ROMILDO PINA AND MAURICIO PIETERZACK
}

\begin{abstract}
In the euclidean space $\left(\mathbb{R}^{n}, g\right)$, with $n \geq 3, g_{i j}=\delta_{i j}$, we consider a (0,4)-tensor $R=T \odot g$ where $T=\sum_{i} f_{i}(x) d x_{i}^{2}$ is a diagonal (0,2)-tensor. We obtain necessary and sufficient conditions for the existence of a metric $\bar{g}$, conformal to $g$, such that $\bar{R}=R$, where $\bar{R}$ is the Riemannian curvature tensor of the metric $\bar{g}$. The solution to this problem is given explicitly for the special cases of the tensor $R$, including a case where the metric $\bar{g}$ is complete on $\mathbb{R}^{n}$. Similar problems are considered for locally conformally flat manifolds. As applications of these results we exhibit explicit examples of metrics $\bar{g}$, conformal to $g$ they are solutions for this problem.
\end{abstract}

\section{INTRODUCTION}

An interesting and very studied problem in geometry is the problem of prescribed scalar curvature. When the scalar curvature is constant and $M$ is a compact manifold this is just the famous Yamabe problem. For more details about the problem of prescribed scalar curvature see [11] and [12]. For noncompact manifolds, several authors have obtained progress in this problem. For more details, see for example [11, [13, [14], [15], [16], [17] and their references.

Another interesting problem that is very studied in present days is the prescribed Ricci curvature equation that can be proposed as follow:

Given a symetric (0,2)-tensor $T$, defined on a manifold $M^{n}, n \geq 3$, does there exist a Riemannian metric $g$ such that Ric $g=T$ ?

When $T$ is nonsingular, that is, its determinant does not vanish, a local solution of the Ricci equation always exists, as it was shown by DeTurck in [1]. When $T$ is singular, but still has constant rank and satisfies certain appropriate conditions, then the Ricci equation also admits local solutions (see [2]). Rotationally symmetric nonsingular tensors were considered in [3] and other results can be found in [2, 4], [5], 6], 7] and [11. Recently, A. Pulemotov in [18] have considered the prescribed Ricci curvature problem in a solid torus.

Pina and Tenenblat have obtained results for this problem considering special classes of tensors $\mathrm{T}$ and conformal metrics (see [8, 9] and their references).

Another problem related with the problem (P1) is Prescribed Curvature Tensor problem, that can be proposed as the following:

Date: August, 2014 and, in revised form,

2010 Mathematics Subject Classification. Primary 53C21; Secondary 35N10.

Key words and phrases. Conformal metric, riemannian curvature tensor, scalar curvature, ricci curvature.

The authors were supported in part by CAPES/PROCAD and FAPEG/GO. 
Given a (0,4)-tensor $R$, defined on a manifold $M^{n}, n \geq 3$, does there exists a Riemannian metric $g$ such that $R_{g}=R$, where $R_{g}$ is the Riemannian curvature tensor of the metric $g$ ?

We observe that solving the problem (P2) is equivalent to solving a nonlinear system of partial differential equations of second order.

There are very few studies in the math literature that deal with this problem. In 1986, DeTurck and Yang in [19 have considered the following problem: Let $R$ be a smooth nondegenerate $(0,4)$-tensor over a 3 -manifold $M$. Then for any $x \in M$ there exists a smooth metric $g$ such that $R_{g}=R$ in a neighborhood of $x$, where $R_{g}$ is the Riemannian curvature tensor of the metric $g$. They also obtain the local existence of metrics such that the Ricci curvature Ric and the scalar curvature $K$ satisfy $R i c_{g}+\lambda K g=Q$ for given $\lambda$ and $Q$ is a symmetric (0,2)-tensor. In 1994, Kowalski and Belger in [20 have considered in $V$ be a $n$ dimensional real vector space, equipped with a scalar product and tensors $K^{(l)}$ over $V$ satisfying all the identities for a Riemannian curvature tensor which satisfies certains inequalities, and the authors have proved that in this case there always exists an analytic Riemannian metric on one open ball such that the derivatives of its curvature tensor are the tensors $K^{(l)}$.

In an attempt to resolve it, we can consider the Prescribed Curvature Tensor problem with change conformal of metric. More precisely, we can consider the following problem:

Let $\left(M^{n}, g\right), n \geq 3$, a Riemannian manifold. Given a (0,4)-tensor $R$, defined on a manifold $M$, does there exist a Riemannian metric $\bar{g}$ conformal to $g$ such that $\bar{R}_{\bar{g}}=R$, where $\bar{R}_{\bar{g}}$ is the Riemannian curvature tensor of the metric $\bar{g}$ ?

The curvature tensor of the metric $g$ on $M$ can be decomposed in

$$
R_{g}=W_{g}+A_{g} \odot g
$$

where $R_{g}$ is the Riemannian curvature tensor, $\odot$ is the Kulkarni-Nomizu product, $A_{g}$ and $W_{g}$ are the Schouten and the Weyl tensors of $g$, respectivelly (see [10]).

Since the Weyl tensor is conformally invariant and especially in the case that $g$ is locally conformally flat $W_{g}=0$, if $g$ is locally conformally flat the Riemann curvature tensor is determined by the Schouten tensor and your decomposition is

$$
R_{g}=A_{g} \odot g .
$$

In this paper, we consider on $\left(\mathbb{R}^{n}, g\right), n \geq 3$, the euclidean space, a $(0,4)$-tensor $R=T \odot g$, where $T$ is a diagonal $(0,2)$-tensor given by $T=\sum_{i} f_{i}(x) d x_{i}^{2}$, with $f_{i}(x)$ are smooth functions. Our interest is to study the Prescribed Curvature Tensor problem in this particular situation.

In Theorem 3.2 we provide necessary and sufficient conditions over the tensor $R$ for the existence of a metric $\bar{g}$ conformal to $g$ such that $\bar{R}_{\bar{g}}=R$. We also extend this result to locally conformally flat manifolds in Theorem 3.8. We consider particular cases for the tensor $R$ when the solutions for the Prescribed Curvature Tensor problem are given explicitilly. In Theorem 3.3 we consider the case in which $R=f(g \odot g)$, for a smooth function $f$ and $g$ is the euclidean metric. Unfortunately, in this case the metric $\bar{g}$ is not complete. In Theorem 3.5 we obtain a result of nonexistence. In Theorem 3.6 we consider tensors $T$ depending on only one fixed 
variable and from this result we exhibit examples of complete metrics on $\mathbb{R}^{n}$ with prescribed Riemannian curvature tensor.

\section{Preliminaires}

Our principal goal is to study the Prescribed Curvature Tensor problem in locally conformally flat manifolds. In this section, we set forth the notation and review the necessary background to state the results about this problem in the next section.

Let $\left(M^{n}, g\right)$, a flat manifold and $R=T \odot g$, where $T$ is a diagonal $(0,2)$-tensor, a $(0,4)$-tensor defined on $M$. We want to find $\bar{g}=\frac{1}{\varphi^{2}} g$ such that $\bar{R}=R$, where $\bar{R}$ is the Riemannian curvature tensor of the metric $\bar{g}$. That is, we want to study the problem

$$
\left\{\begin{array}{l}
\bar{g}=\frac{1}{\varphi^{2}} g \\
\bar{R}=R
\end{array}\right.
$$

Using the decomposition of the Riemannian curvature tensor $\bar{R}$ in (1.1) we obtain that $\bar{R}=R$ is equivalent to $A_{\bar{g}} \odot \bar{g}=T \odot g$. As $\bar{g}=\frac{1}{\varphi^{2}} g$ this equation is equivalent to

$$
A_{\bar{g}} \odot\left(\frac{g}{\varphi^{2}}\right)=T \odot g
$$

Thus, $\bar{R}=R$ is equivalent to

$$
A_{\bar{g}} \odot g=\left(\varphi^{2} T\right) \odot g .
$$

Since the Nomizu-Kulkarni product is injective (see Lemma 1.113 in [10]), the problem (2.1) is equivalent to

$$
\left\{\begin{array}{l}
\bar{g}=\frac{1}{\varphi^{2}} g \\
A_{\bar{g}}=\varphi^{2} T
\end{array}\right.
$$

From now we are considering the euclidean space $\left(\mathbb{R}^{n}, g\right), n \geq 3$, with coordinates $x=\left(x_{1}, . ., x_{n}\right)$ and $g_{i j}=\delta_{i j}$. Given a $(0,4)$-tensor $R=T \odot g$, where $T$ is a diagonal $(0,2)$-tensor given by $T=\sum_{i} f_{i}(x) d x_{i}^{2}$, with $f_{i}(x)$ smooth functions, we seek necessary and sufficient conditions for the tensor $R=T \odot g$ to the existence of a metric $\bar{g}=\frac{1}{\varphi^{2}} g$ such that $\bar{R}=R$.

The Schouten tensor of $\bar{g}$ is defined by

$$
A_{\bar{g}}=\frac{1}{n-2}\left(\operatorname{Ric}_{\bar{g}}-\frac{\bar{K}}{2(n-1)} \bar{g}\right),
$$

where $R i c_{\bar{g}}$ and $\bar{K}$ are the Ricci tensor and the scalar curvature of $\bar{g}$, respectively.

As $\bar{g}$ is conformal to the euclidean metric $g$, the Ricci tensor of $\bar{g}$ is given by

$$
\operatorname{Ric}_{\bar{g}}=\frac{1}{\varphi^{2}}\left\{(n-2) \varphi \text { Hess }_{g} \varphi+\left(\varphi \Delta_{g} \varphi-(n-1)\left|\nabla_{g} \varphi\right|^{2}\right) g\right\}
$$

and the scalar curvature of $\bar{g}$ is given by

$$
\bar{K}=(n-1)\left(2 \varphi \Delta_{g} \varphi-n\left|\nabla_{g} \varphi\right|^{2}\right)
$$


where $\Delta_{g}$ and $\nabla_{g}$ denote the laplacian and the gradient in the euclidean metric $g$, respectivelly (see [10]).

Using the expressions (2.3) and (2.4) the Schouten tensor of $\bar{g}$ can be expressed by

$$
A_{\bar{g}}=\frac{\operatorname{Hess}_{g} \varphi}{\varphi}-\frac{\left|\nabla_{g} \varphi\right|^{2}}{2 \varphi^{2}} g
$$

We will denote by $\varphi_{, x_{k}}$ and $f_{i, x_{k}}$ the derivatives of $\varphi$ and $f_{i}$ with respect to $x_{k}$, respectively, $\varphi_{, x_{i} x_{j}}$ and $f_{i, x_{i} x_{j}}$ the second order derivatives of $\varphi$ and $f$ with respect to $x_{i} x_{j}$, respectively. Since $g$ is the euclidean metric in $\mathbb{R}^{n}, n \geq 3$, studying the

problem (2.2) when $T=\sum_{i} f_{i}(x) d x_{i}^{2}, f_{i}(x)$ are smooth functions, is equivalent to studying the following system of equations

$$
\left\{\begin{array}{l}
\frac{\varphi, x_{i} x_{i}}{\varphi}-\frac{\left|\nabla_{g} \varphi\right|^{2}}{2 \varphi^{2}}=\varphi^{2} f_{i}, \quad \forall \quad i: 1, \ldots, n . \\
\varphi_{, x_{i} x_{j}}=0, \quad \forall \quad i \neq j .
\end{array}\right.
$$

From the second equation of (2.6) it follows that $\varphi$ can be expressed as a sum of functions, each of which depends only on one of the variables $x_{i}$, so we will write $\varphi(x)=\sum_{i=1}^{n} \varphi_{i}\left(x_{i}\right)$.

We will studing the system (2.6) with the additional condition that $3 f_{i}(x)+$ $f_{j}(x) \neq 0$, for all $x \in \mathbb{R}^{n}$ and all $i \neq j$.

\section{MAIN RESULTS}

We now state our main results. We start with a lemma which proves to be very useful in the proofs to follows.

Lemma 3.1. Let $\varphi\left(x_{1}, \ldots, x_{n}\right)$ be a solution of (2.6). Then

$$
\frac{\varphi_{, x_{j}}}{\varphi}=-\frac{f_{i, x_{j}}}{3 f_{i}+f_{j}}, \quad \forall i \neq j
$$

and

$$
\frac{f_{k, x_{j}}}{3 f_{k}+f_{j}}=\frac{f_{i, x_{j}}}{3 f_{i}+f_{j}} .
$$

for distinct $i, j, k$.

Proof. From the first equation of (2.6) we obtain

$$
\varphi_{, x_{i} x_{i}}=\varphi^{3} f_{i}+\frac{\sum_{k}\left(\varphi_{, x_{k}}\right)^{2}}{2 \varphi} .
$$


Taking the derivative with respect to $x_{j}, j \neq i$ and using again the first equation of (2.6) we obtain

$$
\begin{aligned}
0 & =3 \varphi^{2} \varphi_{, x_{j}} f_{i}+\varphi^{3} f_{i, x_{j}}+\frac{2 \varphi_{, x_{j}} \varphi_{, x_{j} x_{j}} 2 \varphi-2 \varphi_{, x_{j}}\left|\nabla_{g} \varphi\right|^{2}}{4 \varphi^{2}} \\
& =3 \varphi^{2} \varphi_{, x_{j}} f_{i}+\varphi^{3} f_{i, x_{j}}+\varphi_{, x_{j}} \frac{\varphi_{, x_{j} x_{j}}}{\varphi}-\frac{\varphi_{, x_{j}}\left|\nabla_{g} \varphi\right|^{2}}{2 \varphi^{2}} \\
& =3 \varphi^{2} \varphi_{, x_{j}} f_{i}+\varphi^{3} f_{i, x_{j}}+\varphi_{, x_{j}} \varphi^{2} f_{j}+\frac{\varphi_{, x_{j}}\left|\nabla_{g} \varphi\right|^{2}}{2 \varphi^{2}}-\frac{\varphi_{, x_{j}}\left|\nabla_{g} \varphi\right|^{2}}{2 \varphi^{2}} \\
& =\varphi^{2}\left(\varphi_{, x_{j}}\left(3 f_{i}+f_{j}\right)+\varphi f_{i, x_{j}}\right)
\end{aligned}
$$

Therefore, since $\varphi \neq 0$ we obtain

$$
\varphi_{, x_{j}}\left(3 f_{i}+f_{j}\right)+\varphi f_{i, x_{j}}=0
$$

and consequently (3.1). The equation (3.2) follows imediatelly from (3.1).

The relationship between the conformal factor $\varphi$ and the functions $f_{i}$ that compose the tensor $T$ in this Lemma is fundamental to establish the results of this work.

The next theorem is our main result and contains necessary and suficient conditions for problem (2.1) to have a solution.

Theorem 3.2. Let $\left(\mathbb{R}^{n}, g\right), n \geq 3$, be the euclidean space, with coordinates $x_{1}, \ldots, x_{n}$, and metric $g_{i j}=\delta_{i j}$. Consider a (0,4)-tensor $R=T \odot g$, where $T=\sum_{i=1}^{n} f_{i}(x) d x_{i}^{2}$, $f_{i}(x)$ are smooth functions such that $3 f_{i}(x)+f_{j}(x) \neq 0$ for all $x \in \mathbb{R}^{n}$ and all $i \neq j$. Then there exists a positive function $\varphi$ such that the metric $\bar{g}=\frac{1}{\varphi^{2}} g$ satisfies $\bar{R}=R$ if and only if the functions $f_{i}$ satisfy the following system of differential equations

$$
\left\{\begin{array}{l}
\frac{f_{i, x_{j}}}{3 f_{i}+f_{j}}=\frac{f_{k, x_{j}}}{3 f_{k}+f_{j}}, \quad i \neq j, \quad k \neq j, \\
\left(\frac{f_{j, x_{i}}}{3 f_{j}+f_{i}}\right)_{, x_{k}}=\left(\frac{f_{j, x_{k}}}{3 f_{j}+f_{k}}\right)_{, x_{i}}, \quad i \neq j, \quad k \neq j, \\
\left(\frac{f_{i, x_{j}}}{3 f_{i}+f_{j}}\right)_{, x_{i}}=\left(\frac{f_{j, x_{i}}}{3 f_{j}+f_{i}}\right)_{, x_{j}}, \quad i \neq j, \\
\frac{1}{2}\left(\frac{f_{j, x_{i}}}{3 f_{j}+f_{i}}\right)^{2}-\left(\frac{f_{j, x_{i}}}{3 f_{j}+f_{i}}\right)_{, x_{i}}-\frac{1}{2} \sum_{k \neq i}\left(\frac{f_{j, x_{k}}}{3 f_{j}+f_{k}}\right)^{2}=h_{i}, \quad i \neq j, \\
\frac{f_{j, x_{i}}}{3 f_{j}+f_{i}} \frac{f_{i, x_{j}}}{3 f_{i}+f_{j}}=\left(\frac{f_{i, x_{j}}}{3 f_{i}+f_{j}}\right)_{, x_{i}}, \quad i \neq j,
\end{array}\right.
$$

where $h_{i}(x)=f_{i} e^{-2 \int \frac{f_{i, x_{j}}}{3 f_{i}+f_{j}} d x_{j}+\psi\left(\hat{x_{j}}\right)}$, the function $\psi\left(\hat{x_{j}}\right)$ does not depend on $x_{j}$, satisfies the following system of $(n-1)$ differential equations

$$
\psi_{, x_{i}}=\int\left(\frac{f_{i, x_{j}}}{3 f_{i}+f_{j}}\right)_{, x_{i}} d x_{j}-\frac{f_{j, x_{i}}}{3 f_{j}+f_{i}}, \quad \text { for } \quad i \neq j
$$


and, up to a multiplicative constant,

$$
\varphi(x)=\exp \left(-\int \frac{f_{i, x_{j}}}{3 f_{i}+f_{j}} d x_{j}+\psi\left(\hat{x_{j}}\right)\right) .
$$

Proof. Suppose $\bar{g}=g / \varphi^{2}$ is a solution of (2.2). Then, by the Lemma 3.1 the first equation of (3.4) is satisfied and we obtain, for a fixed $j=1, \ldots, n$, that

$$
\varphi(x)=\exp \left(-\int \frac{f_{i, x_{j}}}{3 f_{i}+f_{j}} d x_{j}+\psi\left(\hat{x_{j}}\right)\right), \quad i \neq j,
$$

where the function $\psi\left(\hat{x_{j}}\right)$ does not depend on $x_{j}$.

We will show that the expression of $\varphi$ is independent of the variable of integration and that the function $\psi$ is well-defined.

Taking the derivative of $\varphi$ with respect to $x_{i}, i \neq j$ we obtain

$$
\psi_{, x_{i}}=\int\left(\frac{f_{i, x_{j}}}{3 f_{i}+f_{j}}\right)_{, x_{i}} d x_{j}+\frac{\varphi_{, x_{i}}}{\varphi}=\int\left(\frac{f_{i, x_{j}}}{3 f_{i}+f_{j}}\right)_{, x_{i}} d x_{j}-\frac{f_{j, x_{i}}}{3 f_{j}+f_{i}} .
$$

Now, taking the derivative of this expression with respect to $x_{k}, k \neq j$, we obtain

$$
\psi_{, x_{i} x_{k}}=\int\left(\frac{f_{i, x_{j}}}{3 f_{i}+f_{j}}\right)_{, x_{i} x_{k}} d x_{j}-\left(\frac{f_{j, x_{i}}}{3 f_{j}+f_{i}}\right)_{, x_{k}} .
$$

Similarly, we obtain that $\psi_{, x_{k} x_{i}}=\int\left(\frac{f_{i, x_{j}}}{3 f_{i}+f_{j}}\right)_{, x_{k} x_{i}} d x_{j}-\left(\frac{f_{j, x_{k}}}{3 f_{j}+f_{k}}\right)_{, x_{i}}$.

Thus, $\psi_{, x_{i} x_{k}}=\psi_{, x_{k} x_{i}}$ if and only if

$$
\left(\frac{f_{j, x_{i}}}{3 f_{j}+f_{i}}\right)_{, x_{k}}=\left(\frac{f_{j, x_{k}}}{3 f_{j}+f_{k}}\right)_{, x_{i}} .
$$

Therefore, the second equation in (3.4) is satisfied.

Taking the derivative of $\psi_{, x_{i}}$ with respect to $x_{j}, i \neq j$, we obtain

$$
\psi_{, x_{i} x_{j}}=\left(\frac{f_{i, x_{j}}}{3 f_{i}+f_{j}}\right)_{, x_{i}}-\left(\frac{f_{j, x_{i}}}{3 f_{j}+f_{i}}\right)_{, x_{j}} .
$$

Since $\psi_{, x_{i} x_{j}}=\psi_{, x_{j} x_{i}}=0$, the third equation in (3.4) is satisfied.

Using the equation (3.1) we have that, for a fixed $j=1, \ldots, n$,

$$
\ln \varphi(x)=-\int \frac{f_{i, x_{j}}}{3 f_{i}+f_{j}} d x_{j}+\psi\left(\hat{x_{j}}\right), \quad i \neq j,
$$

where the function $\psi\left(\hat{x_{j}}\right)$ does not depend on $x_{j}$.

Integrating the equation 3.1 with respect to another variable $x_{s}$, for a fixed $s=1, \ldots, n, s \neq j$, we obtain

$$
\ln \tilde{\varphi}(x)=-\int \frac{f_{i, x_{s}}}{3 f_{i}+f_{s}} d x_{s}+\tilde{\psi}\left(\hat{x_{s}}\right), \quad i \neq s,
$$

where the function $\tilde{\psi}\left(\hat{x_{s}}\right)$ does not depend on $x_{s}$.

Setting $A=\ln \varphi(x)-\ln \tilde{\varphi}(x)$ we will show that $A$ is constant.

Taking the derivative with respect to $x_{s}$ we obtain

$$
A_{, x_{s}}=-\int\left(\frac{f_{i, x_{j}}}{3 f_{i}+f_{j}}\right)_{, x_{s}} d x_{j}+\psi_{, x_{s}}+\frac{f_{i, x_{s}}}{3 f_{i}+f_{s}}
$$


Using the equation (3.2) and that $\psi_{, x_{s}}=\int\left(\frac{f_{i, x_{j}}}{3 f_{i}+f_{j}}\right)_{, x_{s}} d x_{j}-\frac{f_{i, x_{s}}}{3 f_{i}+f_{s}}$ we conclude that $A_{, x_{s}}=0$. Similarly, taking the derivative of $\stackrel{A}{A}$ with respect to $x_{j}$, using the equation (3.2) and that expression of $\tilde{\psi}_{, x_{j}}$ we conclude that $A_{, x_{j}}=0$.

Now, for $k \neq s$ and $k \neq j$, using the expressions of the derivatives of $\psi$ and $\tilde{\psi}$ and the equation (3.2) from Lemma 3.1 we obtain

$$
\begin{aligned}
A_{, x_{k}}= & -\int\left(\frac{f_{i, x_{j}}}{3 f_{i}+f_{j}}\right)_{, x_{k}} d x_{j}+\psi_{, x_{k}}+\int\left(\frac{f_{i, x_{s}}}{3 f_{i}+f_{s}}\right)_{, x_{k}} d x_{s}-\tilde{\psi}_{, x_{k}} \\
= & -\int\left(\frac{f_{i, x_{j}}}{3 f_{i}+f_{j}}\right)_{, x_{k}} d x_{j}+\int\left(\frac{f_{i, x_{j}}}{3 f_{i}+f_{j}}\right)_{, x_{k}} d x_{j}+\frac{\varphi, x_{k}}{\varphi} \\
& +\int\left(\frac{f_{i, x_{s}}}{3 f_{i}+f_{s}}\right)_{, x_{k}}^{, x_{k}} d x_{s}-\int\left(\frac{f_{i, x_{s}}}{3 f_{i}+f_{s}}\right)_{, x_{k}} d x_{s}-\frac{\tilde{\varphi}, x_{k}}{\tilde{\varphi}} \\
= & -\frac{f_{i, x_{k}}}{3 f_{i}+f_{k}}+\frac{f_{i, x_{k}}}{3 f_{i}+f_{k}}=0 .
\end{aligned}
$$

Thus, $\varphi$ is well-defined. Since $\varphi$ is a solution of (2.2) then $\varphi$ satisfies (2.6). From Lemma 3.1, for $i \neq j$, we have $\varphi_{, x_{i}}=-\varphi \frac{f_{j, x_{i}}}{3 f_{j}+f_{i}}$. Taking the derivative with respect to $x_{i}$, we obtain

$$
\frac{\varphi, x_{i} x_{i}}{\varphi}=-\frac{\varphi_{, x_{i}}}{\varphi} \frac{f_{j, x_{i}}}{3 f_{j}+f_{i}}-\left(\frac{f_{j, x_{i}}}{3 f_{j}+f_{i}}\right)_{, x_{i}}=\left(\frac{f_{j, x_{i}}}{3 f_{j}+f_{i}}\right)^{2}-\left(\frac{f_{j, x_{i}}}{3 f_{j}+f_{i}}\right)_{, x_{i}}
$$

Thus, using that $\left|\nabla_{g} \varphi\right|^{2}=\sum_{k=1}^{n}\left(\varphi_{, x_{k}}\right)^{2}=\sum_{k \neq i} \varphi^{2}\left(\frac{f_{j, x_{k}}}{3 f_{j}+f_{k}}\right)^{2}+\left(\varphi_{, x_{i}}\right)^{2}$ and the expression above, the first equation in (2.6) is equivalent to

$$
\left(\frac{f_{j, x_{i}}}{3 f_{j}+f_{i}}\right)^{2}-\left(\frac{f_{j, x_{i}}}{3 f_{j}+f_{i}}\right)_{, x_{i}}-\frac{1}{2} \sum_{k \neq i}\left(\frac{f_{j, x_{k}}}{3 f_{j}+f_{k}}\right)^{2}-\frac{1}{2}\left(\frac{f_{j, x_{i}}}{3 f_{j}+f_{i}}\right)^{2}=\varphi^{2} f_{i} .
$$

Simplifying this expression, we obtain

$$
\frac{1}{2}\left(\frac{f_{j, x_{i}}}{3 f_{j}+f_{i}}\right)^{2}-\left(\frac{f_{j, x_{i}}}{3 f_{j}+f_{i}}\right)_{, x_{i}}-\sum_{k \neq i}\left(\frac{f_{j, x_{k}}}{3 f_{j}+f_{k}}\right)^{2}=f_{i} e^{\left(-2 \int \frac{f_{i}}{3 f_{i}+f_{j}} d x_{j}+\psi\left(\hat{x_{j}}\right)\right)} .
$$

which proves the fourth equality of (3.4).

From Lemma 3.1, we obtain for $i \neq j$ that

$$
\begin{aligned}
\varphi_{, x_{j} x_{i}} & =-\varphi, f_{i, x_{i}} \frac{f_{i, x_{j}}}{3 f_{i}+f_{j}}-\varphi\left(\frac{f_{i, x_{j}}}{3 f_{i}+f_{j}}\right)_{, x_{i}} \\
& =\varphi\left\{\frac{f_{j, x_{i}}}{3 f_{j}+f_{i}} \frac{f_{i, x_{j}}}{3 f_{i}+f_{j}}-\left(\frac{f_{i, x_{j}}}{3 f_{i}+f_{j}}\right)_{, x_{i}}\right\}=0 .
\end{aligned}
$$

Since $\varphi \neq 0$ the other expression is equal zero, which proves the fifth and last equality of (3.4). The converse is a straightforward computation.

In order to provide explicit examples of metrics satisfying $\bar{R}=R$, we shall consider particular cases for $T$. 
Theorem 3.3. Let $\left(\mathbb{R}^{n}, g\right), n \geq 3$, be the euclidean space, with coordinates $x_{1}, \ldots, x_{n}$, and metric $g_{i j}=\delta_{i j}$. Then there exists a metric $\bar{g}=\frac{1}{\varphi^{2}} g$ such that $\bar{R}=R=$ $f(g \odot g)$, where $f$ is a nonvanishing smooth function, if and only if

$$
f(x)=\frac{-\lambda}{2\left(\sum_{i=1}^{n}\left(a x_{i}^{2}+b_{i} x_{i}\right)+c\right)^{4}}
$$

where $a, b_{i}, c$ are constants, $\lambda=\sum_{i=1}^{n} b_{i}^{2}-4 a c$ and

$$
\varphi(x)=\sum_{i=1}^{n}\left(a x_{i}^{2}+b_{i} x_{i}\right)+c .
$$

Any such metric $\bar{g}$ is unique up to homothety. Moreover, we have:

(1) if $\lambda<0$ then $\bar{g}$ is globally defined on $\mathbb{R}^{n}$;

(2) if $\lambda \geq 0$ then the set of singularities of $\bar{g}$ is

(a) the empty set if $\lambda=0$ and $a=0$;

(b) a point if $\lambda=0$ and $a \neq 0$;

(c) a hyperplane if $\lambda>0$ and $a=0$;

(d) an $(n-1)$-dimensional sphere if $\lambda>0$ and $a \neq 0$.

Proof. Since $f_{i}=f_{j}$, for all $i, j$ from Lemma 3.1 we have

$$
\frac{\varphi, x_{j}}{\varphi}=-\frac{f_{, x_{j}}}{4 f} \text { for all } j \text {. }
$$

Therefore, there exist a constant $\lambda$ such that $\varphi^{4} f=-\frac{\lambda}{2}$ and soon $f=-\frac{\lambda}{2 \varphi^{4}}$.

From (2.6) we have that

$$
\varphi_{, x_{i} x_{i}}=f \varphi^{3}+\frac{\left|\nabla_{g} \varphi\right|^{2}}{2 \varphi}, \quad \text { for all } \quad i=1, \ldots, n .
$$

Then

$$
\varphi_{, x_{i} x_{i}}=\varphi_{, x_{j} x_{j}}
$$

for all $i, j$. Thus, for every $i=1, \ldots, n, \varphi_{i}\left(x_{i}\right)=a x_{i}^{2}+b_{i} x_{i}+c_{i}$ and

$$
\varphi(x)=\sum_{i=1}^{n} \varphi_{i}\left(x_{i}\right)=\sum_{i=1}^{n}\left(a x_{i}^{2}+b_{i} x_{i}\right)+c .
$$

Using the above relation between $\varphi$ and $f$, we obtain that

$$
f(x)=\frac{-\lambda}{2\left(\sum_{i=1}^{n}\left(a x_{i}^{2}+b_{i} x_{i}\right)+c\right)^{4}} .
$$

Calculating the expressions in the first equality in (2.6) we obtain that $\lambda=\sum_{i} b_{i}^{2}-4 a c$. Analyzing the expression of $\varphi$ we arrive to the conclusions concerning the domain of $\varphi$. Particularly, if $\lambda<0$ the function $\varphi$ does not vanish and the metric $\bar{g}$ is globally defined on $\mathbb{R}^{n}$. 
Remark 3.4. In this theorem, although we proved directly from the system (2.6), the equations (3.4) of the Theorem 3.2 are satisfied with $f_{i}=f \forall i=1, \ldots, n$ and $\varphi$ given by (3.7) is the same as in the Theorem 3.2 with $\psi\left(\hat{x_{j}}\right)$ being a constant. This demonstrates that there exist examples of tensor in $\mathbb{R}^{n}$ that are solutions of the equations of Theorem 3.2

Now, we present a result of non-existense of conformal metrics to a special kind of tensor $R$.

Theorem 3.5. Let $\left(\mathbb{R}^{n}, g\right), n \geq 3$, be the euclidean space, with coordinates $x_{1}, \ldots, x_{n}$, and metric $g_{i j}=\delta_{i j}$. Consider the (0,4)-tensor $R=T \odot g$, where $T=\sum_{i=1}^{n} f_{i}\left(x_{i}\right) d x_{i}^{2}$, with $f_{i}\left(x_{i}\right)$ smooth functions that depend on only the variable $x_{i}$ such that $3 f_{i}\left(x_{i}\right)+$ $f_{j}\left(x_{j}\right) \neq 0$ for all $i \neq j$. Then there is no metric $\bar{g}=\frac{1}{\varphi^{2}}$ g such that $\bar{R}=R$.

Proof. Since $f_{i}$ do not depend on the variables $x_{j}, j \neq i$, from (3.1) we have

$$
\frac{\varphi_{, x_{j}}}{\varphi}=-\frac{f_{i, x_{j}}}{3 f_{i}+f_{j}}=0 \text { for all } j \neq i \text {. }
$$

and

$$
\frac{\varphi_{, x_{i}}}{\varphi}=-\frac{f_{j, x_{i}}}{3 f_{j}+f_{i}}=0 \text { for all } i \neq j .
$$

Thus, $\varphi_{, x_{k}}=0$ for all $k$, and therefore $\varphi$ is constant. Using (2.6) we conclude that $f_{i}=0$, for all $i=1, \ldots, n$, which contradicts $3 f_{i}+f_{j} \neq 0$, for $i \neq j$ and therefore does not exist a metric $\bar{g}$ such that $\bar{R}=R$.

In the particular case in which the components of the tensor $T$ depend only one variable, we have the following result.

Theorem 3.6. Let $\left(\mathbb{R}^{n}, g\right), n \geq 3$, be the euclidean space, with coordinates $x_{1}, \ldots, x_{n}$, and metric $g_{i j}=\delta_{i j}$. Consider a (0,4)-tensor $R=T \odot g$ where $T$ is a diagonal (0,2)-tensor given by $T=\sum_{i=1}^{n} f_{i}\left(x_{k}\right) d x_{i}^{2}$, with $f_{i}\left(x_{k}\right)$ smooth functions that depend only on $x_{k}$, for some fixed $k, 1 \leq k \leq n$ such that $3 f_{i}\left(x_{k}\right)+f_{j}\left(x_{k}\right) \neq 0$ for all $i \neq j$. There exists a metric $\bar{g}=\frac{1}{\varphi^{2}} g$ such that $\bar{R}=R$ if and only if all the functions $f_{i}$ for $i \neq k$ are equal to one another, say $f_{i}=f$ for $i \neq k$, and the functions $f$ and $f_{k}$ satisfy the system

$$
\left\{\begin{array}{l}
\frac{1}{2}\left(\frac{f_{, x_{k}}}{3 f+f_{k}}\right)^{2}-\left(\frac{f_{, x_{k}}}{3 f+f_{k}}\right)_{, x_{k}}=C^{2} f_{k} v \\
-\left(\frac{f_{, x_{k}}}{3 f+f_{k}}\right)^{2}=2 C^{2} f v
\end{array}\right.
$$

where $v=v\left(x_{k}\right)=e^{-2 \int \frac{f, x_{k}}{3 f+f_{k}} d x_{k}}$ and if $f_{k}$ and $f$ satisfy these conditions, then $\varphi$ depends only on $x_{k}$ and is given by

$$
\varphi\left(x_{k}\right)=C \exp \left(-\int \frac{f_{, x_{k}}}{3 f+f_{k}} d x_{k}\right)
$$

where $C$ is a positive constant. 
Proof. Since $f_{i}=f_{i}\left(x_{k}\right)$ for some fixed $k$, from the Lemma 3.1 we have that $\varphi_{, x_{j}}=0$ for every $j \neq k$ and therefore $\varphi=\varphi\left(x_{k}\right)$. Furthermore, from the equality in (2.6) we have that $f_{i}=-\frac{\left|\nabla_{g} \varphi\right|^{2}}{2 \varphi^{4}}$ for all $i \neq k$ and, therefore $f_{i}=f_{j}$, if $i \neq k$ and $j \neq k$. From this, the expression of $\varphi$ in (3.12) and the system in (3.11) are consequences of the Theorem 3.2.

Corollary 3.7. Let $\left(\mathbb{R}^{n}, g\right), n \geq 3$, be the euclidean space, with coordinates $x_{1}, \ldots, x_{n}$, and metric $g_{i j}=\delta_{i j}$. Consider the $(0,2)$-tensor

$$
T=f_{k}\left(x_{k}\right) d x_{k}^{2}+f\left(x_{k}\right) \sum_{i \neq k} d x_{i}^{2},
$$

where $f_{k}\left(x_{k}\right)=\frac{h^{2}-2 h_{, x_{k}}}{2 C^{2}} e^{2 \int h\left(x_{k}\right) d x_{k}}, f\left(x_{k}\right)=-\frac{h^{2}}{2 c^{2}} e^{2 \int h\left(x_{k}\right) d x_{k}}$, with $h=h\left(x_{k}\right)$ a smooth function that depends only on $x_{k}$, for some fixed $k, 1 \leq k \leq n$. Then there exist a conformal metric $\bar{g}=g / \varphi^{2}$ such that $\bar{R}=R$ and

$$
\varphi\left(x_{k}\right)=C \exp \left(-\int h\left(x_{k}\right) d x_{k}\right),
$$

where $C$ is a positive constant.

If, in addition, $0 \leq\left|\int h\left(x_{k}\right) d x_{k}\right| \leq L$, for a finite constant $L$, then the metric $\bar{g}$ is complete on $\mathbb{R}^{n}$.

Proof. Follows immediately from Theorem 3.6 considering $h\left(x_{k}\right)=\frac{f_{, x_{k}}}{3 f+f_{k}}$. The equalities in (3.11) are trivially satisfied and the expressions of $f$ and $f_{k}$ are exactly the components of the tensor $T$.

We can extend the Theorem 3.2 to locally conformally flat manifolds. Consider $\left(M^{n}, g\right)$, a Riemannian manifold locally conformally flat. We may consider the problem (2.1) for neighborhood $V \subset M$, with local coordinates $\left(x_{1}, x_{2}, \ldots, x_{n}\right)$ such that $g_{i j}=\delta_{i j} / F^{2}$, where $F$ is a nonvanishing smooth function on $V$.

Theorem 3.8. Let $\left(M^{n}, g\right), n \geq 3$, be Riemannian manifold, locally conformally flat. Let $V$ be an open subset of $M$ with coordinates $x=\left(x_{1}, x_{2}, \ldots, x_{n}\right)$ with $g_{i j}=$ $\delta_{i j} / F^{2}$. Consider a $(0,4)$-tensor $R=T \odot g$, where $T$ is a diagonal $(0,2)$-tensor given by $T=\sum_{i=1}^{n} f_{i}(x) d x_{i}^{2}$, with $f_{i}$ are smooth functions such that $3 f_{i}(x)+f_{j}(x) \neq 0$ for all $x \in V$ and all $i \neq j$. Then there exists a metric $\bar{g}=\frac{1}{\phi^{2}} g$ such that $\bar{R}=R$ if and only if the functions $f_{i}, \varphi$ and $\psi$ are given as in Theorem 3.2 and $\phi=\frac{\varphi}{F}$.

Proof. We consider $\varphi=\phi F$ and apply Theorem 3.2

Remark 3.9. In a similar fashion, we can extend the Theorem 3.6 for locally conformally flat manifolds.

As an application of the Theorem 3.8 we show that given a certain $(0,4)$-tensor $R$ in $\mathbb{R}_{+}^{n}$ there exist a metric $\bar{g}$, conformal to the metric of the hyperbolic space whose Riemannian curvature tensor is $R$. 
Example 3.10. Let $\mathbb{H}^{n}=\left(\mathbb{R}_{+}^{n}, g\right)$ the hyperbolic space, where $g=\frac{1}{x_{n}^{2}} g_{0}$, with $\left(g_{0}\right)_{i j}=\delta_{i j}$ the euclidean metric and $\mathbb{R}_{+}^{n}=\left\{x=\left(x_{1}, x_{2}, \ldots, x_{n}\right) \in \mathbb{R}^{n} / x_{n}>0\right\}$.

Given the (0,4)-tensor $R=T \odot g$, where $T$ is the diagonal (0,2)-tensor

$$
T=-\frac{\left(2 x_{n}^{2}-1\right)^{2}}{2 x_{n}^{4}} e^{2 x_{n}^{2}} \sum_{i \neq n} d x_{i}^{2}+\frac{4 x_{n}^{4}-8 x_{n}^{2}-1}{2 x_{n}^{4}} e^{2 x_{n}^{2}} d x_{n}^{2}
$$

defined in $\mathbb{R}_{+}^{n}$, Theorem 3.8 garantees the existence of a metric $\bar{g}=\frac{1}{\phi^{2}} g$, where $\phi(x)=\frac{\varphi(x)}{F(x)}=e^{-x_{n}^{2}}$ such that $\bar{R}=R$.

Moreover, since $\phi(x)$ is bounded, $\left(\mathbb{R}_{+}^{n}, \bar{g}\right)$ is a complete Riemannian manifold, conformal to the hyperbolic space.

The scalar curvature of $\left(\mathbb{R}_{+}^{n}, \bar{g}\right)$ is not constant and given by

$$
\bar{K}=(n-1) e^{-2 x_{n}^{2}}\left(4(2-n) x_{n}^{4}+4(n-3) x_{n}^{2}-n\right)
$$

and the Ricci tensor of the metric $\bar{g}$ is

$$
R i c_{\bar{g}}=\frac{4(2-n) x_{n}^{4}+2(2 n-5) x_{n}^{2}+1-n}{x_{n}^{2}} \sum_{i \neq n} d x_{i}^{2}+(n-1) \frac{4 x_{n}^{4}-4 x_{n}^{2}-1}{x_{n}^{2}} d x_{n}^{2} .
$$

Likewise, $\left(\mathbb{R}_{+}^{n}, \bar{g}\right)$ does not have constant sectional curvature, given by

$$
K\left(\frac{\partial}{\partial x_{i}}, \frac{\partial}{\partial x_{j}}\right)=-\left(1-2 x_{n}^{2}\right)^{2} e^{-2 x_{n}^{2}} \leq 0
$$

if $i, j \neq n$ and

$$
K\left(\frac{\partial}{\partial x_{i}}, \frac{\partial}{\partial x_{n}}\right)=2 x_{n}^{2}\left(2 x_{n}^{2}-3\right) e^{-2 x_{n}^{2}} .
$$

Example 3.11. Corollary 3.7 also provide examples of complete, conformally flat manifolds with prescribed Riemannian curvature tensor and non-constant curvatures.

(1) In the euclidean space $\left(\mathbb{R}^{n}, g\right), n \geq 3$, consider the $(0,4)$-tensor $R=T \odot g$, where $T$ is a diagonal $(0,2)$-tensor given by

$$
T=\left(\frac{\sinh ^{2} x_{k}-2 \cosh x_{k}}{2 C^{2}} e^{2 \cosh x_{k}}\right) d x_{k}^{2}-\frac{\sinh ^{2} x_{k}}{2 C^{2}} e^{2 \cosh x_{k}} \sum_{i \neq k} d x_{i}^{2},
$$

where $C$ is a positive constant.

Corollary 3.7 garantees the existence of a metric $\bar{g}=\frac{1}{\varphi^{2}} g$, conformal to the euclidean metric, such that $\bar{R}=R=T \odot g$ is the Riemannian curvature tensor of the metric $\bar{g}$. In particular, we have that

$$
\varphi\left(x_{k}\right)=C e^{-\cosh x_{k}}
$$

where $C$ is a positive constant. The manifold $\left(\mathbb{R}^{n}, \bar{g}\right)$ is complete, has negative scalar curvature given by

$$
\bar{K}=-(n-1) C e^{-\cosh x_{k}}\left(2 \cosh ^{2} x_{k}+(n-2) \sinh ^{2} x_{k}\right)
$$


and negative Ricci curvature whose Ricci tensor is negative definite and given by

$$
\operatorname{Ric}_{\bar{g}}=-(n-1) \cosh x_{k} d x_{k}^{2}-\left(\cosh x_{k}+(n-2) \sinh ^{2} x_{k}\right) \sum_{i \neq k} d x_{i}^{2} .
$$

Moreover, $\left(\mathbb{R}^{n}, \bar{g}\right)$ has nonpositive sectional curvature given by

$$
K\left(\frac{\partial}{\partial x_{i}}, \frac{\partial}{\partial x_{j}}\right)=-C^{2} \sinh ^{2} x_{k} e^{-2 \cosh x_{k}}
$$

if $i, j \neq k$ and

$$
K\left(\frac{\partial}{\partial x_{i}}, \frac{\partial}{\partial x_{k}}\right)=-C^{2} \cosh x_{k} e^{-2 \cosh x_{k}} .
$$

(2) In the euclidean space $\left(\mathbb{R}^{n}, g\right), n \geq 3$, consider the $(0,4)$-tensor $R=T \odot g$, where $T$ is a diagonal $(0,2)$-tensor given by

$$
T=\frac{\left(4 x_{k}^{2}-2\right)}{C^{2}} d x_{k}^{2}-\frac{2 x_{k}^{2}}{C^{2}} \sum_{i \neq k} d x_{i}^{2},
$$

where $C$ is a positive constant. Corollary 3.7 garantees the existence of a metric $\bar{g}=\frac{1}{\varphi^{2}} g$, conformal to the euclidean metric, such that $\bar{R}=R=T \odot g$ is the Riemannian curvature tensor of the metric $\bar{g}$. In particular, we have that

$$
\varphi\left(x_{k}\right)=\frac{C}{1+x_{k}^{2}}
$$

where $C$ is a positive constant. The manifold $\left(\mathbb{R}^{n}, \bar{g}\right)$ is complete, has negative scalar curvature given by

$$
\bar{K}=-\frac{4(n-1) C^{2}}{\left(1+x_{k}^{2}\right)^{2}}\left(1+(n-3) x_{k}^{2}\right)
$$

and the Ricci tensor of $\bar{g}$ is given by

$$
R i c_{\bar{g}}=\frac{2(n-1)\left(x_{k}^{2}-1\right)}{\left(1+x_{k}^{2}\right)^{2}} d x_{k}^{2}+\frac{(10-4 n) x_{k}^{2}-2}{\left(1+x_{k}^{2}\right)^{2}} \sum_{i \neq k} d x_{i}^{2} .
$$

Moreover, $\left(\mathbb{R}^{n}, \bar{g}\right)$ has sectional curvature given by

$$
K\left(\frac{\partial}{\partial x_{i}}, \frac{\partial}{\partial x_{j}}\right)=-\frac{4 C^{2} x_{k}^{2}}{\left(1+x_{k}^{2}\right)^{4}}
$$

if $i, j \neq k$ and

$$
K\left(\frac{\partial}{\partial x_{i}}, \frac{\partial}{\partial x_{k}}\right)=-\frac{2 C^{2}\left(1-x_{k}^{2}\right)}{\left(1+x_{k}^{2}\right)^{4}} .
$$

(3) In the euclidean space $\left(\mathbb{R}^{n}, g\right)$ consider the $(0,4)$-tensor $R=T \odot g$, where $T$ is a diagonal $(0,2)$-tensor given by

$$
T=\frac{2\left(x_{k}^{2}-1\right)}{C^{2}} e^{2 x_{k}^{2}} d x_{k}^{2}-\frac{2 x_{k}^{2}}{C^{2}} e^{2 x_{k}^{2}} \sum_{i \neq k} d x_{i}^{2},
$$

where $C$ is a positive constant. Corollary 3.7 garantees the existence of a metric $\bar{g}=\frac{1}{\varphi^{2}} g$, conformal to the euclidean metric, such that $\bar{R}=R=T \odot g$ 
is the Riemannian curvature tensor of the metric $\bar{g}$. In particular, we have that

$$
\varphi\left(x_{k}\right)=C e^{-x_{k}^{2}}
$$

where $C$ is a positive constant. The manifold $\left(\mathbb{R}^{n}, \bar{g}\right)$ is complete, has negative scalar curvature given by

$$
\bar{K}=-4(n-1) C^{2} e^{-2 x_{k}^{2}}\left(1+(n-2) x_{k}^{2}\right)
$$

and negative Ricci curvature, whose Ricci tensor is given by

$$
\operatorname{Ric}_{\bar{g}}=-2(n-1) d x_{k}^{2}-2\left(1-2(n-2) x_{k}^{2}\right) \sum_{i \neq k} d x_{i}^{2}
$$

The sectional curvature of $\left(\mathbb{R}^{n}, \bar{g}\right)$ is nonpositive and given by the expressions

if $i, j \neq k$ and

$$
K\left(\frac{\partial}{\partial x_{i}}, \frac{\partial}{\partial x_{j}}\right)=-4 x_{k}^{2} C^{2} e^{-2 x_{k}^{2}}
$$

$$
K\left(\frac{\partial}{\partial x_{i}}, \frac{\partial}{\partial x_{k}}\right)=-2 C^{2} e^{-2 x_{k}^{2}}
$$

We observe that although there are points where the tensor $R$ is zero, there still exists a complete metric such that the curvature tensor of this metric is the prescribed tensor $R$.

\section{REFERENCES}

1. D. DeTurck, Existence of metrics with prescribed Ricci Curvature: Local Theory, Invent. Math. 65 (1981), 179-207.

2. D. DeTurck and H. Goldschmidt, Metrics with Prescribed Ricci Curvature of Constant Rank, Advances in Mathematics 145, 1-97.

3. J. Cao and D. DeTurck, The Ricci curvature equation with rotational symmetry, American Journal of Mathematics 116 (1994), 219-241.

4. D. DeTurck, Metrics with prescribed Ricci curvature, Seminar on Differential Geometry, Ann. of Math. Stud. 102, (S. T. Yau, ed.), Princeton University Press (1982), 525-537.

5. D. DeTurck and W. Koiso, Uniqueness and non-existence of metrics with prescribed Ricci curvature, Ann. Inst. H. Poincaré Anal. Non Linéaire 1 (1984), 351-359.

6. R. S. Hamilton, The Ricci curvature equation, Seminar on nonlinear partial differential equations (Berkeley, California)(1983), 47-72.

7. J. Lohkamp, Metrics of negative Ricci curvature, Ann. Math. 140 (1994), 655-683.

8. R. Pina and K. Tenenblat, Conformal Metrics and Ricci Tensors on the Sphere. Proc. Amer. Math. Soc. 132 (2004), 3715- 3724.

9. R. Pina and K. Tenenblat, On solutions of the Ricci curvature equation and the Einstein equation. Israel J. Math. 171 (2009), 61-76.

10. A. L. Besse, Einstein Manifolds, Ergeb. Math. Grenzgeb. (3) 10, Springer, Berlin, 1987.

11. T. Aubin, Some nonlinear problems in Riemannian geometry, Springer-Verlag, Berlin, 1998.

12. K.-S. Cheng and J.-L. Chern, Conformal metrics with prescibed curvature on $S^{n}$, Journal of Differential Equations 116 (1993), 155-185.

13. Z. Jin, Prescribing scalar curvatures on the conformal classes of complete metrics with negative curvature, Trans. Amer. Math. Soc. 340 (1993), 785-810.

14. X. Ma and R. C. McOwen, Complete conformal metrics with zero scalar curvature, Proc. Amer. Math. Soc. 115 (1992), 69-77.

15. K.-S. Cheng and W.-M. Ni, On the struture of the conformal scalar curvature equation on $\mathbb{R}^{n}$, Indiana Univ. Math. J. 41 (1992), 261-278.

16. J. L. Kazdan and F. W. Warner, Scalar curvature and conformal deformation of Riemannian structure, J. Differential Geometry 10 (1975), 113-134. 
17. J. Bland and M. Kalka, Negative scalar curvature metrics on noncompact manifolds, Trans. Amer. Math. Soc. 316 (1989), 433-446.

18. A. Pulemotov, Metrics with prescribed Ricci curvature near the boundary of a manifold, Math. Ann. 357 (2013), no. 3, 969-986.

19. D. DeTurck and D. Yang, Local existence of smooth metrics with prescribed curvature, Nonlinear problems in geometry (Mobile, Ala., 1985), Contemp. Math., 51, Amer. Math. Soc., Providence, RI, (1986), 37-43.

20. O. Kowalski and M. Belger, Riemannian metrics with the prescribed curvature tensor and all its covariant derivatives at one point, Math. Nach. 168 (1994), 209-225.

Instituto de Matemática e Estatística, Universidade Federal de Goiás, Goiânia, BRASIL, 74001-970

Current address: Insituto de Matemática e Estatística, Universidade Federal de Goiás, Goiânia, Brasil, 74001-970

E-mail address: romildo@ufg.br

Instituto de Matemática e Estatística, Universidade Federal de Goiás, Goiânia, BRASIL, 74001-970

E-mail address: mauriciopieterzack@gmail.com 